\title{
Psicooncología
}

ISSN: 1696-7240

\section{Terapias artísticas y creativas en la mejora del malestar psicológico en mujeres con cáncer de mama. Revisión sistemática}

\author{
Jennifer Rabadán ${ }^{1}$, Andrés Chamarro ${ }^{2 *}$ y María Álvarez ${ }^{3}$
}

Recibido: 15 de agosto de 2017 / Aceptado: 25 de agosto de 2017

Resumen: Objetivo: Conocer el estado actual de la eficacia de las terapias artísticas y creativas (TAC) en las variables de malestar psicológico de mujeres con cáncer de mama y delimitarla en función del tipo de TAC y momento terapéutico del paciente. Método: La búsqueda se realizó mediante las bases de datos Pubmed, Web of Science, Psychinfo y Google Academic para el periodo 2010-2016. Resultados: Se obtienen 9 estudios RTC y cuasi-experimentales controlados (2 de Danza movimiento terapia, 1 de Arte Terapia, 1 de Musicoterapia y 5 de Escucha musical) que incluyen 680 participantes. Se discute la calidad metodológica, el riesgo de sesgo y las medidas de eficacia terapéutica. Conclusiones: Las TAC parecen ser una ayuda complementaria a corto plazo durante la fase de tratamiento en cáncer de mama. Sin embargo, son necesarios más ensayos para aclarar los mecanismos subyacentes a la mejora, así como delimitar la eficacia de cada modalidad.

Palabras clave: Cáncer de mama, terapia artística, terapia creativa, musicoterapia, terapia con música, danza movimiento terapia, arte terapia.

\section{[en] Artistic and creative therapies in improving psychological distress in women with breast cancer. Systematic review}

\begin{abstract}
Objective: To know the current state of the effectiveness of artistic and creative therapies (ACTs) over psychological distress of women with breast cancer and to delimit it according to the type of $\mathrm{CT}$ and the therapeutic moment of the patient. Method: The search was performed using Pubmed, Web of Science, Psychinfo and Google Academic databases for the period 2010-2016. Results: nine controlled RTC and quasi-experimental studies (2 of Dance movement therapy, 1 of Art Therapy, 1 of Music therapy and 5 of Musical Listening) were obtained, including 680 participants. Methodological quality, risk of bias and measures of therapeutic efficacy are discussed. Conclusions: ACT scans seem to be short-term complementary aid during the treatment phase in breast cancer. However, more trials are needed to clarify the mechanisms underlying the improvement as well as to delimit the effectiveness of each modality.

Keywords: Breast cancer, creative therapy, artistic therapy, music therapy, dance movement therapy, art therapy.
\end{abstract}

1 Departamento de Psicología Básica, Evolutiva y de la Educación. Universitat Autònoma de Barcelona. 08193 Bellaterra (Cerdanyola del Vallès)

E-mail: jennifer.rgonzalez.14@gmail.com

2 Departamento de Psicología Básica, Evolutiva y de la Educación Universidad Autónoma de Barcelona. 08193

- Bellaterra (Cerdanyola del Vallès).

E-mail: andres.chamarro@uab.cat

3 Departamento de Psicología Básica, Evolutiva y de la Educación. Universidad Autónoma de Barcelona. 08193

- Bellaterra (Cerdanyola del Vallès).

E-mail: maria.alvarez@uab.cat

* Dirección de correspondencia: Andrés Chamarro. (autor para correspondencia) Departamento de Psicología Básica, Evolutiva y de la Educación Universidad Autónoma de Barcelona 08193 - Bellaterra (Cerdanyola del Vallès). E-mail: andres.chamarro@uab.cat 
Sumario. 1. Introducción 2. Método 3. Resultados 4. Discusión 5. Conclusiones 6. Referencias bibliográficas.

Cómo citar: Rabadán J, Chamarro A, Álvarez M. Terapias artísticas y creativas en la mejora del malestar psicológico en mujeres con cáncer de mama. Revisión sistemática. Psicooncología 2017;14:187-202. Doi: 10.5209/PSIC.57079.

\section{Introducción}

Se estima que una de cada ocho mujeres se sitúa en riesgo de padecer cáncer de mama a lo largo de su vida ${ }^{(1)}$. En 2012, la Sociedad Española de Oncología Médica ${ }^{(2)}$ situaba el cáncer de mama como el tipo de cáncer más prevalente $(28,99 \%)$ entre la población femenina. Al final del año, se diagnosticaron 25.215 casos nuevos y las cifras de mortalidad se igualaron a 6.075. Sin embargo, la supervivencia se ha ido incrementado año tras año llegando a un $82,8 \%{ }^{(3)}$.

Por lo que respecta al proceso de intervención, a lo largo de sus diferentes fases (diagnóstico, tratamiento, intervalo libre, supervivencia, recidiva y fase final de la vida) los pacientes se enfrentan a un escenario complejo que incluye las secuelas orgánicas, estéticas, funcionales y cognitivas de las intervenciones médicas (mastectomía, quimioterapia, radioterapia, etc.), así como consecuencias en el entorno social y familiar ${ }^{(4)}$. Dicha situación amenazante, cambiante y persistente en el tiempo da lugar a reacciones de estrés que implican la activación de estrategias de afrontamiento y adaptación constantes ${ }^{(5)}$.

Recogiendo estas problemáticas, las autoridades sanitarias destacan la importancia de tratar las necesidades psicosociales, de rehabilitación y reinserción social de los pacientes. Esto se ha traducido en el interés por la mejora de la calidad de vida ${ }^{(6)}$, de la que las afectaciones psicológicas, físicas y sociolaborales forman parte ${ }^{(7)}$. El aumento de la incidencia y la supervivencia demanda un esfuerzo continuado por mejorar y garantizar la mejor calidad de vida de los pacientes.

La fatiga, el dolor, el estrés, la ansiedad y la depresión son los síntomas más prevalentes en la población con cáncer de mama. Un estudio reciente ${ }^{(8)}$ identifica una elevada prevalencia de fatiga durante (49\%), al finalizar (47\%) y un año posterior (31\%) a la intervención de quimioterapia. La prevalencia más baja se identificó después de la cirugía (9\%). Por otro lado, la prevalencia del dolor persistente después del tratamiento se sitúa entre el $22 \%$ y el $53 \%{ }^{(9)}$. Otros estudios recientes apuntan a que un 38,3\% de mujeres experimentan dolor a los 3 años posteriores a una mastectomía ${ }^{(10)}$. Por lo que respecta al estrés, éste aparece entre un $70-77 \%$ de las mujeres en la fase de diagnóstico reciente, según varios estudios ${ }^{(11)}$. Finalmente, según el estudio de Burgess ${ }^{(12)}$, alrededor del $50 \%$ de las mujeres presentan depresión o ansiedad posterior al año de diagnóstico. Con el tiempo disminuyen al $25 \%$ en el segundo y tercer año, hasta llegar al 15\% en el cuarto año.

Las intervenciones en el cáncer de mama que muestran eficacia en los síntomas expuestos se desglosan en: a) Intervenciones informativo-educativas, como psicoeducación, couselling y autoayuda; b) Intervenciones psicológicas individuales, que incluyen técnicas conductuales, cognitivas, terapia cognitivoconductual, terapia breve pre-operativa, mindfulness o técnicas creativas y artísticas; y c) Intervenciones grupales de expresión emocional y apoyo, psicoeducacional o 
cognitivo-conductual ${ }^{(5)}$. La mayoría de las intervenciones cuentan con numeras investigaciones que avalan su eficacia, sin embargo, las terapias artísticas y creativas (TAC) cuentan con escasa investigación ${ }^{(13)}$. Dichas técnicas utilizan la creación artística (pintura, escultura, danza, teatro, música, fotografía) como herramienta terapéutica. En ellas el terapeuta establece una relación y un espacio donde el paciente utiliza el lenguaje artístico con el fin de facilitar la expresión, comunicación, reflexión, autoconocimiento, afrontamiento y desarrollo personal ${ }^{(14)}$. Se puede practicar en sesiones individuales o en pequeños grupos. En oncología son usadas para manejar los síntomas vinculados al tratamiento y facilitar el proceso de reajuste psicológico en las diversas etapas de la enfermedad ${ }^{(13)}$.

Existen diversas modalidades de estas técnicas. El arte terapia consiste en el uso de materiales plástico-visuales para la creación de obras plásticas, desde las más clásicas (dibujo, pintura, escultura, estampación) a las más actuales o modernas (assemblage, collage, fotografía, video, body-art, performances) ${ }^{(15)}$. La terapia a través de la música es la modalidad más estudiada en pacientes con cáncer ${ }^{(16)}$. Existen dos tipos de intervenciones con música. La musicoterapia o music therapy, incluye un proceso terapéutico sistemático (evaluación, tratamiento, valoración) e individualizado por un músico terapeuta capacitado y el uso de experiencias musicales adaptadas, tales como escuchar música, tocar música con un instrumento, improvisar música y componer. Por otro lado, la medicina musical o music medicine consiste en la escucha de música pregrabada ofrecida por un profesional médico o sanitario. Finalmente, la modalidad de la danza movimiento terapia (DMT) se basa en la interacción entre el cuerpo y la mente, trabajando principalmente con el movimiento, la emoción y el lenguaje corporal ${ }^{(17)}$. La DMT promueve la expresión espontánea y creativa del movimiento con el objetivo de que aparezcan pensamientos y sentimientos que son a menudo difíciles de articular en palabras y promueve el apoyo interpersonal. La DMT permitiría descargar las emociones y la tensión psicológica y tomar conciencia de fortalezas personales y recursos internos. Se sugiere en el paciente oncológico para adquirir mayor autoeficacia, aumentar la conciencia corporal, mejora la autoimagen, aceptar los cambios corporales y mejorar el estado de ánimo.

Actualmente el arte terapia ${ }^{(18)}$, la musicoterapia ${ }^{(19)}$ y la DMT $^{(20)}$ son las TAC más investigadas en cáncer en general, y en cáncer de mama en particular ${ }^{(21)}$. Una reciente revisión ${ }^{(22)}$ sugiere mejoras con respecto a ansiedad y depresión, calidad de vida, afrontamiento, estrés, ira y estado de ánimo. Sin embargo, aparecen contradicciones dado que otras sugieren mejoras exclusivamente en ansiedad, pero no en calidad de vida, depresión y otros factores como el dolor, estado anímico y afrontamiento ${ }^{(21)}$. Ambas coinciden en la necesidad de realizar más y mejores investigaciones de calidad. Por otro lado, debido a la heterogeneidad de cada tipo de técnica, los efectos no son generalizables a todas las TAC por igual. Paralelamente, están apareciendo centros y asociaciones que incluyen las TAC en su amplio abanico de intervenciones.

El interés científico y práctico de las TAC en la mejora de la calidad de vida de las mujeres con cáncer de mama demanda evidencia, así como delimitaciones entre los tipos de terapia y sus efectos. Por ello, los objetivos de la presente revisión tratan de evaluar y sintetizar el estado de la eficacia de las TAC en variables de malestar psicológico de mujeres con cáncer de mama y delimitarla en función de la intervención artística y creativa y el momento terapéutico. 


\section{Método}

La presente revisión sigue los estándares y recomendaciones PRISMA para la planificación, desarrollo y redacción de las revisiones sistemáticas ${ }^{(23,24,26)}$.

\subsection{Criterios de selección de los estudios}

Los estudios seleccionados incluyeron:

1. Mujeres con cáncer de mama en cualquier estadio o fase de la enfermedad y sin padecer disfunción física o cognitiva grave.

2. Intervenciones basadas exclusivamente en TAC (uso de la música, musicoterapia, uso de la danza, danza movimiento terapia, uso de pintura, escultura, etc.)

3. Variables de malestar psicológico como variables resultado. Se incluyen a) Variables psicológicas (depresión, ira, ansiedad, estrategias de afrontamiento, calidad de vida etc.), b) Variables físicas (fatiga, náuseas, dolor, calidad del sueño), c) Variables fisiológicas (frecuencia cardiaca, frecuencia respiratoria, etc.), d) Variables de apoyo social, espiritual y de comunicación (verbalización, gestos, etc.).

4. Información sobre la modalidad de intervención oncológica (cirugía, radioterapia o quimioterapia), curso clínico (diagnóstico, tratamiento, intervalo libre, recidiva, remisión, etc.) y el momento temporal (antes, durante o después) del tratamiento oncológico respecto la aplicación de la variable tratamiento (TAC).

5. Ensayos controlados experimentales o cuasi experimentales.

6. Artículos completos, tesis doctorales y resúmenes de congreso.

Se excluyeron:

1. Revisiones, meta-análisis, artículos teóricos, estudios observacionales y estudios de caso.

2. Estudios en idiomas diferentes al castellano o inglés.

\subsection{Estrategias de búsqueda}

Las bases de datos electrónicas empleadas para la búsqueda sistemática de literatura de la presente revisión corresponden a: Pubmed, Web of Science, Psychinfo y Google Academic. Se usaron las palabras clave "breast cancer", "art therapy" "art", "dance", "music", "drawing", "painting"y "dance movement therapy". El periodo de búsqueda se estableció entre enero de 2011 y diciembre de 2016, dado que ya existen revisiones previas a este periodo. Adicionalmente se añadieron estudios previos a 2011 que no estaban incluidos en la revisión previa ${ }^{(21)}$ y que cumplían los criterios de inclusión mencionados previamente. La búsqueda se inició en octubre de 2016 y finalizó en diciembre de 2016. El algoritmo de búsqueda relacionó mediante el operador booleano "AND" el tipo de población propuesta "Breast cancer" con los conceptos relacionados con las posibles intervenciones propuestas ("Art therapy" OR "Art" OR "Dance" OR "Music" OR "Draw*" OR "Paint*” OR "Dance Movement Therapy"). Finalmente se estableció una búsqueda alternativa en Google Academic para tesis o disertaciones utilizando algoritmos similares a los mencionados previamente. 


\subsection{Proceso de selección}

En la búsqueda mediante la base de datos se localizaron 118 documentos (115 artículos y 3 disertaciones), de los cuales se excluyeron 25 por estar duplicados y 60 por no cumplir los criterios de inclusión. El proceso continuó con la lectura de los resúmenes restantes, 20 de los cuales no se correspondían con los criterios de inclusión de forma clara. Los 13 restantes requerían una revisión más exhaustiva y se incluyeron en archivo Excel para un análisis detallado. Finalmente, los documentos válidos para la inclusión representaron un total de 11 (7 artículos completos, 2 resúmenes de congresos y 2 tesis doctorales) pasando a formar parte del estudio. Para obtener una visión detallada del proceso de selección ver figura 1.

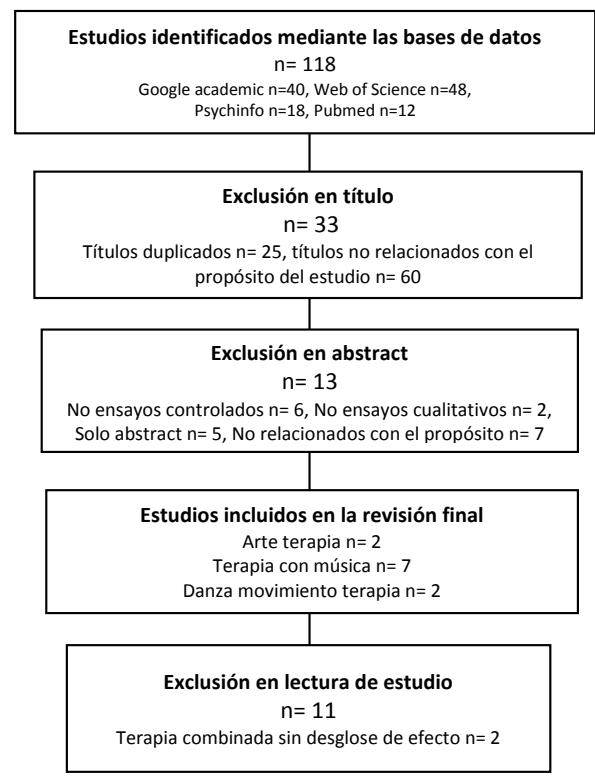

Figura 1. Proceso de selección de los artículos

\subsection{Extracción de datos}

La extracción y volcado de datos fue realizada por el primer autor. Aquellos estudios que cumplieron las medidas de inclusión formaron parte del resumen de los datos. Se extrajo la información relativa al apellido del primer autor, el año de publicación, el tipo de terapia, el diseño del estudio, características de la muestra (n total, edad media total, $\mathrm{n}$ por grupo, intervención individual o grupal), características del profesional que aplica la terapia, la modalidad de tratamiento oncológico y/o curso clínico, el momento temporal del mismo cuando se aplica la TAC, las variables dependientes y los resultados finales. Para ver la información completa véase la tabla 1.

Se consultó a un segundo y tercer investigador para resolver discrepancias y se establecieron criterios de uniformidad tales como la priorización de medidas de comparación entre grupos y la homogenización de la unidad de medida. Únicamente tres estudios muestran el tamaño del efecto a través de la $d$ de Cohen. Por ello, los 


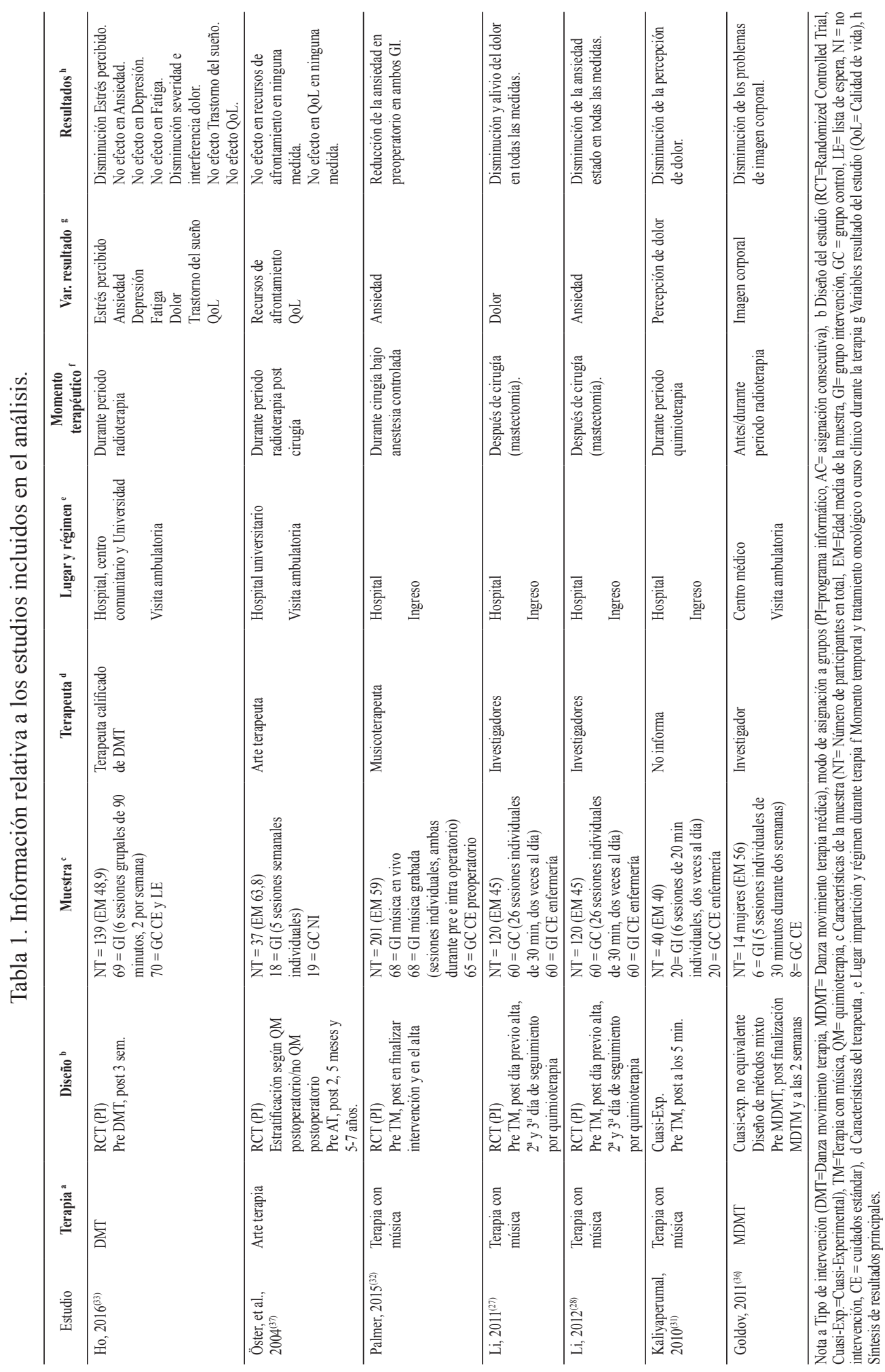




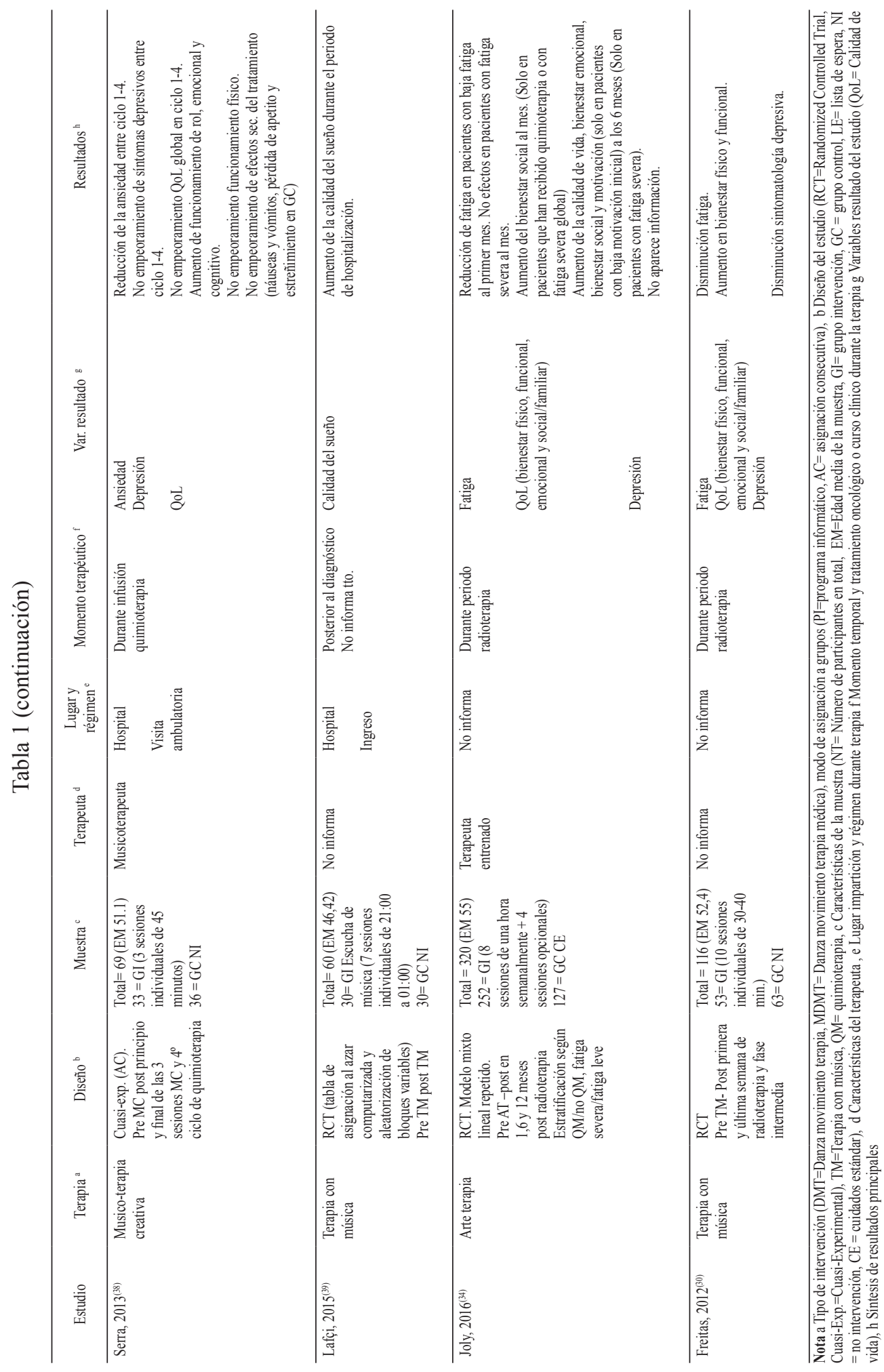


resultados de la eficacia se describen principalmente a efectos de significación ( $p$ $<0,05)$. Adicionalmente se han detallado extensamente las características, variables y resultados de todos los estudios.

\subsection{Evaluación del riesgo de sesgo}

La evaluación del riesgo de sesgo de los estudios se llevó a cabo mediante las directrices de la última versión del Manual Cochrane 5.1.0 ${ }^{(26,27)}$. Los seis dominios evaluados corresponden al sesgo de selección, sesgo de realización, sesgo de detección, sesgo de desgaste, sesgo de información y otros sesgos. Para la evaluación de la calidad los autores adoptaron el rol de jueces. En la figura 2 se describe la valoración del riesgo de sesgo en cada uno de los artículos mediante tres indicadores (bajo riesgo, riesgo no claro y alto riesgo).

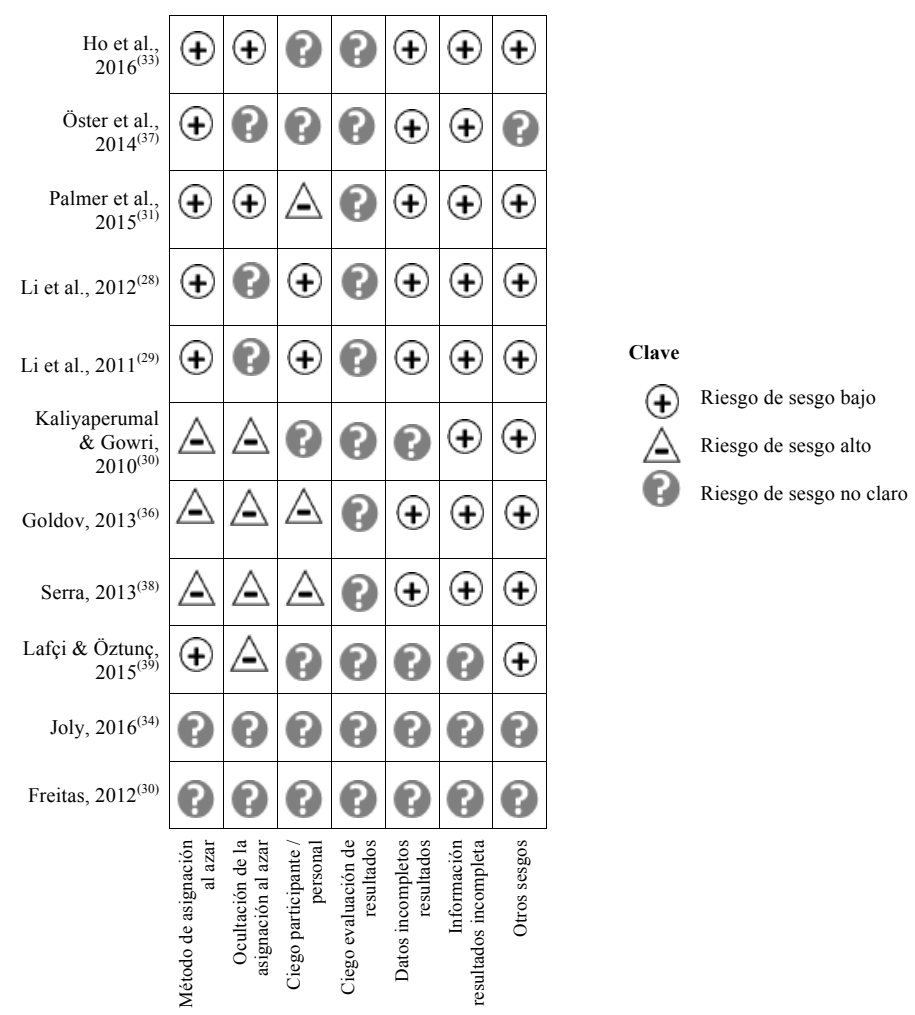

Figura 2. Resumen del riesgo de sesgo: juicio de los revisores sobre el riesgo de sesgo de cada estudio incluido.

\section{Resultados}

\subsection{Estadísticos descriptivos}

Dos de los artículos seleccionados ${ }^{(28,29)}$ pertenecen a una misma población por lo que se tratará como uno solo a efectos de descriptivos y como dos en las variables de 
resultado. El origen de las investigaciones se distribuye en Francia $(n=1)$, Brasil $(n=1)$, Turquía $(n=1)$, Suecia $(n=1)$, EEUU $(n=2)$, China $(n=2)$, India $(n=1)$ y España $(n=1)$.

\subsection{Resultados preliminares}

La muestra cuenta con un total de 1.116 mujeres participantes y se observan poblaciones que varían de 14 a 320 participantes. El rango de edad va de 18 a 94 años con una media total de 51,7 años. Se incluyen exclusivamente mujeres con cáncer de mama, excepto en los estudios de Freitas ${ }^{(30)}$ y Kaliyaperumal y Gowri ${ }^{(31)}$ que también incluyen cáncer ginecológico. En cuanto al curso clínico, las terapias se han llevado a cabo en la fase de tratamiento (cirugía, quimioterapia y radioterapia). Un $20 \%$ de los estudios aplican la terapia durante $(n=1)$ o después $(n=1)$ de la cirugía, un $20 \%$ la ejecutan durante la infusión $(n=1)$ o periodo $(n=1)$ de quimioterapia, un $50 \%(n=5)$ durante el periodo de radioterapia y, finalmente, uno de los estudios se realiza post diagnóstico sin especificar claramente el contexto de tratamiento.

\subsection{Características de la intervención}

Todos los estudios tienen una única condición de intervención excepto el de Palmer et al. ${ }^{(32)}$ que presenta dos condiciones: por un lado, la escucha de música en vivo durante el preoperatorio y música grabada durante la operación y por otro la escucha de música grabada durante el preoperatorio y música grabada durante la operación.

El desglose según terapia, indica que un $20 \%(n=2)$ de los artículos corresponden a arte terapia, un $20 \%(\mathrm{n}=2)$ pertenecen a terapias basadas en DMT y finalmente, un $60 \%(n=6)$ equivale a medicina musical y musicoterapia $(n=5 \mathrm{y} \mathrm{n}=1$ respectivamente $)$.

Las aplicaciones de las intervenciones se han hecho de forma individual para cada usuario excepto en el estudio de Ho et al. ${ }^{(33)}$ que se realizaba en sesiones grupales de 6 a 10 mujeres. Solo un estudio no aporta información ${ }^{(35)}$. Respecto al sujeto que aplica la intervención, las sesiones de DMT, arte terapia y musicoterapia fueron llevadas a cabo por especialistas cualificados de cada especialidad mientras que las sesiones de medicina musical mostraron más diversidad (investigador $n=1$, músicoterapeuta $n=1$, no se informa $n=2$ ).

Las variables resultado de los estudios correspondieron con las variables psicológicas depresión ( 3 estudios), ansiedad $n=4$, estrés $n=1$, calidad de vida $n=5$, recursos de afrontamiento $n=1$, imagen corporal $n=1$, y con las variables físicas (náuseas, vómitos, pérdida de apetito y estreñimiento $n=1$; fatiga $n=3$; dolor $n=3$; calidad del sueño $n=2$ ). No aparecen variables fisiológicas, de comunicación y de apoyo espiritual. Uno de los estudios de calidad de vida utilizaba el apoyo social como indicador de efecto.

La duración y frecuencia de las terapias difiere según la modalidad. Así, en los dos estudios de $\mathrm{DMT}^{(33,36)}$ la frecuencia corresponde a dos o más sesiones semanales con una total de 6 y 5 sesiones y la duración varia de 30 a $90 \mathrm{~min}$. Los estudios de arte terapia ${ }^{(35,37)}$ coinciden en sesiones ( 5 y 8 respectivamente) de una hora semanal. En cuanto al único estudio de musicoterapia ${ }^{(38)}$ éste se distribuye en 3 sesiones de 45 minutos. Por último, en las terapias basadas en la escucha de música ${ }^{(28-31,39)}$, las sesiones oscilan de 20-40 min a 4 horas y se aplican una o dos veces al día entre 3 y 7 días con excepción del estudio de Palmer $^{(32)}$, que no especifica la duración.

En lo referente a la actividad de los grupos control, esta se distribuye en recibir cuidados estándar de los profesionales $(54,5 \%, \mathrm{n}=6)$, estar en lista de espera y recibir cuidados estándar $(9,9 \%, n=1)$ y no recibir la intervención $(36,3 \%, n=4)$. 
Si se exploran las características de cada tipología, se encuentran las siguientes particularidades. Los estudios de DMT coinciden en el uso de la improvisación por medio de movimientos, y en la exploración emocional a través de estos. Ho et al. ${ }^{(33)}$

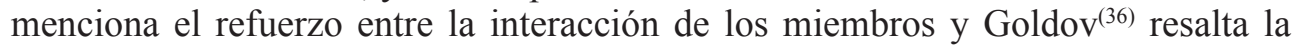
flexibilidad y orientación a las necesidades del paciente en la relación terapeutapaciente. El estudio de musicoterapia ${ }^{(38)}$ se basa en el modelo humanista de Nordoff que establece un enfoque activo donde el paciente crea e improvisa música por medio de distintos instrumentos $\mathrm{y}$, por otro lado, se minimiza la comunicación verbal entre terapeuta y paciente. En el estudio de arte terapia se describe el material utilizado en todas las sesiones (hojas de papel, papel en rollo, acuarelas, pinceles, etc.), así como una breve descripción de las actividades de cada sesión (dibujar imágenes que representan emociones, pintarse el contorno real del cuerpo, actividades a elección del paciente, recopilación de las obras y creación de una imagen resumen final). La mayor variabilidad se encuentra en la aplicación de la escucha musical. La mayoría de pacientes usan auriculares y reproductores digitales, excepto un estudio que usa un sistema de música central en las habitaciones donde el paciente está ingresado ${ }^{(39)}$ y otro que genera música en directo en una de los grupos de intervención ${ }^{(31)}$. El estilo musical es variado (música con arpa, música china, de relajación) y recomendado por profesionales o asociaciones cualificadas. En dos estudios los pacientes seleccionan las piezas musicales entre un repertorio seleccionado ${ }^{(28,29,32)}$.

\subsection{Calidad de la evidencia}

La calidad metodológica para los estudios seleccionados se considera moderada-baja (ver figura 2). Un 66,6\% de los estudios detallan bien el método de aleatorización. Sin embargo el resto $(33,3 \%)$ presentan un diseño cuasi experimental, de manera que existe posibilidad de sesgos debido a posibles diferencias de la muestra no detectadas o al proceso de reclutamiento. Se destaca un porcentaje elevado de alto riesgo $(44,4 \%)$ y riesgo no claro $(33,3 \%)$ respecto la ocultación de la asignación, de manera que la calidad en esta clasificación es baja. Además, en el 77,7\% de los estudios no se aplicó el cegamiento a los evaluadores y participantes o no se reportó dada la dificultad que plantea por las propias características de la intervención. La mayoría de los estudios muestran los datos completos y suficientemente especificados, excepto un $22,2 \%$. Un $65 \%$ de los estudios no reporta ningún tipo de valor o coeficiente sobre el tamaño de la eficacia del tratamiento, sin embargo, el 100\% reporta la significación.

\subsection{Resultados de la eficacia}

Se describen a continuación los principales hallazgos de los estudios revisados desglosados en función del tipo de terapia. En cada apartado se detallan los efectos obtenidos según el momento temporal (antes, durante, después) del tipo de tratamiento (cirugía, quimioterapia, radioterapia), si se indica.

\subsection{Danza movimiento terapia}

Los dos estudios seleccionados se enfocan en pacientes que van a ser sometidos a radioterapia o bien la han iniciado. El estudio de Ho et al. ${ }^{(33)}$ muestran efectos moderados-bajos en reducción del estrés percibido, severidad del dolor e interferencia 
del dolor $(\mathrm{d}=0,34-0,36, \mathrm{p}<, 05)$ al finalizar la intervención grupal (tres semanas). Además, aporta un modelo de estimación de las trayectorias para estas tres variables que muestran una disminución de los niveles en el grupo DMT y un incremento en el grupo control. En el segundo estudio ${ }^{(36)}$, las sesiones individuales de DMT revelan mejoras en la imagen corporal con un tamaño del efecto alto en las fases de seguimiento (BIS: $d=0,5,0,86$; SKIP: $d=0,97,0,31$; BIRS: $d=0,86$ ).

\subsection{Arte terapia}

Un estudio de Arte terapia muestra los resultados del seguimiento a 5 y 7 años de mujeres que la habían recibido durante el periodo de radioterapia. A diferencia del primer seguimiento ${ }^{(40,41)}$, que reporta mejoras de estilo de afrontamiento basado en el apoyo social a los 6 meses, no aparecen efectos en recursos de afrontamiento y calidad de vida a largo plazo.

\subsection{Musicoterapia}

La revisión solo cuenta con un estudio de musicoterapia ${ }^{(38)}$ que se llevó a cabo durante la infusión de quimioterapia de los pacientes. Los resultados muestran reducción de la ansiedad y depresión entre ciclo 4 y 1 , pero no mejora en calidad de vida. Sin embargo, se produce un empeoramiento en la calidad de vida del grupo control en todas las medidas de seguimiento (ciclo 1 al 4). La fatiga empeora en ambos grupos.

\subsection{Terapia con música}

Los estudios que evalúan la escucha de música en el proceso de cirugía corresponden a las investigaciones de Palmer $^{(32)}$ y Li et al. ${ }^{(28,29)}$. El primero evalúa la eficacia durante la intervención preoperatoria e intraoperatoria. Los resultados muestran que ambos grupos de intervención redujeron la ansiedad preoperatoria respecto al grupo control con un tamaño del efecto medio (medida del efecto $=0,57,0,55$ ). Ambas intervenciones no muestran diferencias entre sí. El segundo estudio indica los resultados en aplicar la escucha de música posterior a la cirugía (mastectomía). Los resultados indican reducción significativa de la ansiedad y el dolor respecto al grupo control en las variables ansiedad estado, dolor total, intensidad del dolor y gravedad del dolor. La valoración del dolor total también muestra mejoras significativas en el índice de recuento de palabras en las medidas post. Otro estudio que valora la escucha de música ${ }^{(31)}$ muestra que la percepción de dolor en pacientes que se están sometiendo a quimioterapia se reduce en los tres días de la intervención. Por último, los resultados del estudio de Lafçi y Öztunç ${ }^{(39)}$ indican mejoras en la calidad del sueño al finalizar la intervención (una semana posterior).

\section{Discusión}

La presente revisión pretendía: 1) Evaluar y sintetizar el estado de la eficacia de las TAC en variables de malestar psicológico de mujeres con cáncer de mama, y 2) Valorar los efectos en función del momento terapéutico del paciente con cáncer de mama. En general, aparecen mejoras en el ajuste psicosocial a corto plazo, así 
como disminución del empeoramiento cuando se aplican las TAC. En concreto se muestra reducción en la ansiedad, depresión, calidad de vida, estrés, imagen corporal y síntomas secundarios al tratamiento y reducción del empeoramiento de la calidad de vida y síntomas secundarios al tratamiento oncológico. Si los efectos se valoran en función del momento terapéutico, es decir, el momento temporal (antes, durante o después) del tratamiento oncológico en el que es aplicada la TAC, se encuentra que: a) Durante el periodo de radioterapia, la DMT se muestra eficaz en la reducción de estrés, dolor, fatiga ( corporal. b) En periodo de quimioterapia se reduce la percepción de dolor con la escucha musical; durante la infusión de quimioterapia, mediante la musicoterapia, se disminuye la sintomatología ansiosa y se evita empeoramiento en la calidad de vida, sintomatología depresiva y efectos secundarios del tratamiento (náuseas, vómitos, pérdida de apetito y estreñimiento). c) Durante la intervención quirúrgica, la escucha musical reduce la ansiedad y posterior a ella se reduce la ansiedad y dolor. Un hallazgo adicional contempla que, fuera del momento terapéutico: d) En pacientes que ya han sido diagnosticados, escuchar música antes de ir a dormir mejora la calidad del sueño. e) Mediante el arte terapia no aparecen efectos a largo plazo en recursos de afrontamiento y calidad de vida.

En general, las distintas modalidades de TAC coinciden en la mejora de aspectos como la percepción de dolor, estrés o ansiedad. Sin embargo, no es posible generalizar la aplicación de cualquier modalidad a cualquier momento terapéutico. Según la presente revisión, la DMT es más usada en los periodos de radioterapia. Esto podría explicarse por el empleo del movimiento y el cuerpo, que podría ser contraindicado en otros momentos donde los síntomas físicos son más intensos. Por otro lado, la terapia con música se lleva a cabo principalmente durante la intervención quirúrgica o infusión de quimioterapia en pacientes hospitalizados, posiblemente debido a que es una actividad compatible con las limitaciones impuestas por la terapéutica. Para el arte terapia, la falta de criterios específicos en la literatura no permite establecer el momento adecuado de aplicación. En conjunto, escasean explicaciones relativas a los mecanismos por los cuales se dan los efectos terapéuticos en el contexto del cáncer de mama. Hasta ahora, se han propuesto procesos generales que subyacen a las TAC, como la expresión no verbal de pensamientos y sentimientos difíciles de verbalizar, un entorno de socialización, los procesos de creación e improvisación, la estimulación emocional, cognitiva y somatosensorial, la focalización de la atención en procesamientos específicos de la información y las emociones ${ }^{(42)}$.

Nosotros consideramos que algunos de estos procesos generales, en el caso del cáncer, podrían concretarse en evocación de recuerdos positivos, ventilación emocional, atención a emociones positivas y distracción. En todo caso no podemos determinar cuáles de estos mecanismos se corresponden con las diferentes TAC. A esto hay que añadir que no se dispone de información suficiente respecto a la generalización del uso de estas técnicas entre todas las afectadas por el cáncer de mama.

Hasta ahora la terapia con música es la que contiene mayor investigación y aceptación en la intervención con cáncer de mama ${ }^{(43,44)}$, sin embargo aparecen muy pocos estudios de DMT y arte terapia.

Respecto a investigaciones previas, los resultados sobre DMT parecen aportar luz sobre lo expuesto en revisiones anteriores ${ }^{(20)}$. A diferencia de esta última, sí que aparece mejora en la percepción de dolor, la imagen corporal y el estrés, en este caso en pacientes sometidos a un proceso de radioterapia. Los resultados contradictorios 
podrían explicarse dado que en los estudios de las revisiones previas no se restringía o valoraba por separado los pacientes según el proceso o momento terapéutico. Las intervenciones con arte terapia durante el periodo de radioterapia no parecen ser efectivas a largo plazo en la mejora de recursos de afrontamiento en el dominio social y calidad de vida, a diferencia de la eficacia mostrada en los primeros seguimientos del mismo estudio ${ }^{(40,41)}$. Los resultados referentes a la reducción de ansiedad y percepción del dolor mediante las terapias con música (musicoterapia y escucha musical) son consistentes con las revisiones anteriores ${ }^{(19)}$ y la revisión para cáncer en general ${ }^{(22)}$.

Por último, la mejora de la calidad del sueño en sesiones de escucha musical ha sido poco explorada por lo que no se encuentran revisiones previas.

A diferencia de la revisión previa ${ }^{(21)}$, la actual incluye las características de las muestras descritas en términos de momento terapéutico del paciente. A pesar de la emergencia de nuevas tipologías basadas en la adición de otras técnicas (i.e., relajación progresiva, visualización de imágenes) o combinación con otras terapias, como es el caso del Mindful Movement ${ }^{(45,46)}$, no se han incluido en el análisis para evitar relaciones espurias. Esto puede ayudar a explicar la aparente falta de evolución en la investigación del uso de las TAC en el cáncer de mama. Parece que el interés de los investigadores se desplaza hacia el uso de terapias combinadas.

La evaluación metodológica ha revisado 6 poténciales dominios de riesgo de sesgo, a diferencia de los 3 dominios que evaluaba la revisión previa ${ }^{(21)}$. Los estudios revisados poseen una calidad metodológica entre moderada y baja, por lo que concluimos que existe riesgo en la atribución de causalidad. Así, dada la evaluación de resultados autoinformados, y el escaso control aplicado a la asignación de grupos, personal y participantes, los resultados deben tomarse con precaución.

\subsection{Limitaciones}

Las limitaciones de la presente revisión aluden a la diversidad de variables de malestar psicológico y momentos terapéuticos contemplados, cosa que dificulta extraer conclusiones o aportar claridad. Otra limitación es que para la mayoría de las TAC (DMT, musicoterapia, arte terapia) no se especifica un modelo y protocolo específicos de intervención. La frecuencia y duración de las diferentes intervenciones es variable para cada una de las modalidades. Es decir, las intervenciones no están sistematizadas ni estandarizadas, hecho que dificulta la comparación clara entre ellas y la generalización de la eficacia. Por otro lado, queda por determinar los mecanismos que explican la mejora, así como la existencia de explicación causal de la misma.

\subsection{Futuras investigaciones}

Para futuras investigaciones se propone determinar los mecanismos subyacentes a cada una de las TAC en pacientes con cáncer de mama. Por otro lado, es necesario establecer protocolos estándar que permitan la comparación y mejoren la evidencia.

Referente a la investigación de aplicaciones de TAC, se recomiendan estudios controlados y aleatorizados, que contemplen el ciego en los evaluadores (en los participantes y los terapeutas se requiere la interacción paciente-terapeuta) y que incluyan ensayos con intervenciones grupales, ya que son escasas en la literatura. 


\section{Conclusiones}

Las terapias artísticas y creativas parecen ser una ayuda complementaria a corto plazo durante las fases de tratamiento del cáncer de mama. La presente revisión muestra que las TAC contribuyen a disminuir o bien evitar el empeoramiento del ajuste psicosocial durante los periodos de cirugía, quimioterapia y radioterapia. Sin embargo, son necesarios más ensayos para aclarar los mecanismos subyacentes a la mejora, así como delimitar la eficacia de cada modalidad.

\section{Referencias bibliográficas}

1. Asociación Española Contra el Cáncer, 2016 [Acceso 7 de septiembre de 2017] Disponible en: https://www.aecc.es/SobreElCancer/CancerPorLocalizacion/CancerMama/ Paginas/incidencia.aspx

2. Las Cifras del Cáncer en España. Sociedad Española de Oncología Médica. Madrid. 2016. [Acceso 15 de agosto de 2017]. Disponible en: http://www.seom.org/es/prensa/el-canceren-espanyacom/105941-las-cifras-del-cancer-en-espana-2017

3. Asociación Española Contra el Cáncer, 2017 [Acceso 7 de septiembre de 2017] Disponible en: https://www.aecc.es/SobreElCancer/CancerPorLocalizacion/CancerMama/Paginas/ pronostico.aspx

4. Rojas-may G. Estrategias de intervención psicológica en pacientes con cáncer de mama. Rev Med (Puebla). 2006;17:194-7.

5. García ALG, Hurtado AG, Aranda BE. Eficacia de la terapia cognitivo conductual en mujeres con cáncer de mama. Psicooncología 2015; 12:129-40. doi. 10.5209/rev_PSIC.2015.v12. n1.48908

6. Ministerio de Sanidad y Política Social. Estrategía en Cáncer del Sistema Nacional de Salud. MinisteriodeSanidadyPolíticaSocial2010.[Acceso7deseptiembrede2017]Disponibleen:http:// www.msps.es/organizacion/sns/planCalidadSNS/pdf/ActualizacionEstrategiaCancer.pdf

7. HernándezM, Cruzado JA, PradoC, RodríguezE. Salud mental y malestaremocional en pacientes con cáncer. Psicooncología 2013;9:233-57. doi: 10.5209/rev_PSIC.2013.v9.n2-3.40895

8. Fabi A, Falcicchio C, Giannarelli D, Maggi G, Cognetti F, Pugliese P. The course of cancer related fatigue up to ten years in early breast cancer patients: What impact in clinical practice? Breast 2017;34:44-52. doi:10.1016/j.breast.2017.04.012

9. Mejdahl MK, Andersen KG, Gartner R, Kroman N, Kehlet H. Persistent pain and sensory disturbances after treatment for breast cancer: six year nationwide follow-up study. BMJ 2013;346:1865. doi:10.1136/bmj.f1865

10. Juhl AA, Christiansen P, Damsgaard TE. Persistent pain after breast cancer treatment: a questionnaire-based study on the prevalence, associated treatment variables, and pain type. J Breast Cancer 2016;19:447-54. doi: 10.4048/jbc.2016.19.4.447

11. Mertz BG, Bistrup PE, Johansen C, Dalton SO, Deltour I, Kehlet H, et al. Psychological distress among women with newly diagnosed breast cancer. Eur J Oncol Nurs 2012;16:43943. doi:10.1016/j.ejon.2011.10.001

12. Burgess, C. Depression and anxiety in women with early breast cancer: five-year observational cohort study. BMJ 2005, 330:702. doi: org/10.1136/bmj.38343.670868.D3

13. MJ W, Molassiotis A, Payne S. What research evidence is there for the use of art therapy in the management of symptoms in adults with cancer? A systematic review. Psychooncology 2011;20:135-45 11p. doi: 10.1002/pon.1722.

14. Dieguez M. Creación Artística y Enfermedad Mental. Madrid. 2006. Tesis doctoral [Acceso 16 de agosto de 2017]. Disponible en: http://biblioteca.ucm.es/tesis/bba/ucm-t29445.pdf 
15. López MartínezM. Técnicas, materiales y recursos utilizados en los procesos arteterapéuticos. Arteterapia Papeles arteterapia y Educación artística para la inclusión Social 2011;6: 18391. doi:10.5209/rev_ARTE.2011.v6.37092

16. Martí-Augé P, Mercadal-Brotons M, Solé-Resano C. La musicoterapia en Oncología. Gac Mex Oncol 2015;14:346-52. doi:10.1016/j.gamo.2015.11.013

17. Rodríguez S. Danza Movimiento Terapia: Cuerpo, Psique y Terapia. Av en Salud Ment Relac Rev Int. 2009;8:1-20. [Acceso 12 de agosto]. Disponible en: http://www.psiquiatria. com/bibliopsiquis/assetstore/13/90/39/139039798933210743133017539769177171458

18. Collete N. Arteterapia y cáncer. Psicooncología 2011;8:81-99. doi: 10.5209/rev_ PSIC.2011.v8.n1.7

19. Bradt J, Dileo C, Grocke D, Magill L. Music interventions for improving psychological and physical outcomes in cancer patients. Cochrane Database Syst Rev 2016;(8):CD006911. doi: 10.1002/14651858.CD006911.pub3.

20. Bradt J, Shim M, Goodill SW. Dance/movement therapy for improving psychological and physical outcomes in cancer patients. Cochrane Database Syst Rev 2015;1:CD007103. doi: 10.1002/14651858.CD007103.pub3.

21. Boehm K, Cramer H, Staroszynski T, Ostermann T. Arts therapies for anxiety, depression, and quality of life in breast cancer patients: A systematic review and meta-analysis. EvidenceBased Complement Altern Med 2014; Article ID 103297-doi:10.1155/2014/103297

22. Archer S, Buxton S, Sheffield D. The effect of creative psychological interventions on psychological outcomes for adult cancer patients: A systematic review of randomised controlled trials. Psychooncology 2015; 24:1-10. doi: 10.1002/pon.3607

23. Liberati A, Altman DG, Tetzlaff J, Mulrow C, Gøtzsche PC, Ioannidis JPA, et al. The PRISMA statement for reporting systematic reviews and meta-analyses of studies that evaluate health care interventions: explanation and elaboration. BMJ 2009; 339. doi: 10.1136/bmj.b2700

24. Moher D, Shamseer L, Clarke M, Ghersi D, Liberati A, Petticrew M, et al. Preferred reporting items for systematic review and meta-analysis. BMJ 2015; 349. doi: 10.1136/bmj.g7647

25. Perestelo-Perez L. Standards on how to develop and report systematic reviews in psychology and health. Int J Clin Health Psychol 2013;13:49-57. doi:10.1016/S1697-2600(13)70007-3

26. Higgins JPT, Altman DG, Gøtzsche PC, Jüni P, Moher D, Oxman AD, et al. The Cochrane Collaboration's tool for assessing risk of bias in randomised trials. BMJ 2011; 343. doi: 10.1136/bmj.d5928

27. Centro Cochrane Iberoamericano, T. (2012). Manual Cochrane de Revisiones Sistemáticas de Intervenciones, versión 5.1. 0. [Acceso 10 de agosto de 2017]. Disponible: https:// es.cochrane.org/sites/es.cochrane.org/files/public/uploads/Manual_Cochrane_510_reduit.pdf

28. Li XM, Yan H, Zhou KN, Dang SN, Wang DL, Zhang YP. Effects of music therapy on pain among female breast cancer patients after radical mastectomy: results from a randomized controlled trial. Breast Cancer Res Treat 2011;128:411-9. doi: 10.1007/s10549-011-1533-Z.

29. Li X-M, Yan H, Zhou K-N, Dang S-N, Wang D-L, Zhang Y-P, et al. Effects of music therapy on anxiety of patients with breast cancer after radical mastectomy: a randomized clinical trial. J Adv Nurs 2012; 68:1145-55. doi: 10.1111/j.1365-2648.2011.05824.x

30. Freitas NMA, Silva TRMA, Freitas-Junior R, Paula Junior W, Silva DJ, Machado GDP, et al. Music therapy reduces radiotherapy-induced fatigue in patients with breast or gynecological cancer: a randomized trial. Cancer Res 2012; 72 (24 Supplement), P2-12-04.

31. Kaliyaperumal R, Gowri J. Effect of music therapy for patients with cancer pain. Int J Biol Med Res 2010;3:79-81. 
32. Palmer JB, Lane D, Mayo D, Schluchter M, Leeming R, Palmer JB, et al. Effects of music therapy on anesthesia requirements and anxiety in women undergoing ambulatory breast surgery for cancer diagnosis and treatment : a randomized controlled trial. J Clin Oncol 2015;33:3162-8. doi: 10.1200/JCO.2014.59.6049

33. Ho RTH, Fong TCT, Cheung IKM, Yip PSF, Luk MY. Effects of a short-term dance movement therapy program on symptoms and stress in patients with breast cancer undergoing radiotherapy: a randomized, controlled, single-blind trial. J Pain Symptom Manage 2016;51:824-31. doi:10.1016/j.jpainsymman.2015.12.332

34. Joly F, Pasquier, D, Hanzen, C, Heutte N, Levy C, Le Tinier F, et al. Impact of art therapy (AT) on fatigue and quality of life (QoL) during adjuvant external beam irradiation (EBI) in breast cancer patients (pts): a randomized trial. Ann Oncol 2016; 27 suppl_6:1439PD. doi. org/10.1093/annonc/mdw390.07

35. Molasiotis A. Complementary therapies in cancer: a global perspective. EONS Magazine, 2016; 26-28. [Acceso 25 de septiembre de 2017]Disponible en: https://www.cancernurse. eu/documents/magazine/2016Spring/EONSMagazine2016Spring.pdf

36. Goldov NB. The effects of individualized brief medical dance/movement therapy on body image in women with breast cancer. [Acceso 10 de agosto de 2017]. Tesis doctoral disponible en: http://pqdtopen.proquest.com/doc/1021376710.html?FMT=AI

37. Öster I, Tavelin B, Egberg Thyme K, Magnusson E, Isaksson U, Lindh J, et al. Art therapy during radiotherapy - A five-year follow-up study with women diagnosed with breast cancer. Arts Psychother 2014;41:36-40. doi: 10.1080/07399332.2015.1017640

38. Serra M. Comparación del efecto de una intervención con musicoterapia para mujeres con cáncer de mama durante la sesión de quimioterapia: un análisis cuantitativo y cualitativo. 2013;237. Disponible en: http:/www.tesisenred.net/bitstream/handle/10803/108962/ TESIS MIREIA SERRA VILA_URL.pdf?sequence=3

39. Lafçi D, Öztunç G. The effect of music on the sleep quality of breast cancer patients. Int J Caring Sci 2015;8:633-40.

40. Öster I, Svensk a C, Magnusson E, Thyme KE, Sjõdin M, Åström S, et al. Art therapy improves coping resources: a randomized, controlled study among women with breast cancer. Palliat Support Care 2006;4:57-64.

41. Svensk AC, Öster I, Thyme KE, Magnusson E, SjÖdin M, Eisemann M, et al. Art therapy improves experienced quality of life among women undergoing treatment for breast cancer: A randomized controlled study. Eur J Cancer Care (Engl) 2009;18:69-77. doi: 10.1111/j.1365-2354.2008.00952.x.

42. Lusebrink VB. Art therapy and the brain: an attempt to understand the underlying processes of art expression in therapy. Art Ther 2011;21:125-35. doi:10.1080/07421656.2004.10129496

43. Hole J, Hirsch M, Ball E, Meads C. Music as an aid for postoperative recovery in adults: a systematic review and meta-analysis. Lancet 2015; 386:1659-71. doi:10.1016/S01406736(15)60169-6

44. Greenlee H, DuPont-Reyes MJ, Balneaves LG, Carlson LE, Cohen MR, Deng G, et al. Clinical practice guidelines on the evidence-based use of integrative therapies during and after breast cancer treatment. CA Cancer J Clin 2017;67:194-232. doi: 10.3322/caac.21397.

45. Lesiuk T. the effect of mindfulness-based music therapy on attention and mood in women receiving adjuvant chemotherapy for breast cancer: a pilot study. Oncol Nurs Forum 2015;42:276-82. doi: 10.1188/15.ONF.276-282.

46. Jang SH, Kang SY, Lee HJ, Lee SY. Beneficial effect of mindfulness-based art therapy in patients with breast cancer-a randomized controlled trial. Explore 2016;12:333-340. doi: 10.1016/j.explore.2016.06.003 